\title{
Acute Kidney Injury Following Chimeric Antigen Receptor T-Cell Therapy for B-Cell Lymphoma in a Kidney Transplant Recipient
}

\author{
Edoardo Melilli, Alberto Mussetti, Gabriela Sanz Linares, Marco Ruella, Charette La Salette, \\ Alexandre Savchenko, Maria del Rosario Taco, Nuria Montero, Josep Grinyo, Alex Fava, \\ Montse Gomà, Maria Meneghini, Anna Manonelles, Josepmaria Cruzado, Ana Sureda, and \\ Oriol Bestard
}

\begin{abstract}
Anti-CD19 chimeric antigen receptor (CAR) T-cell therapy is a newer and effective therapeutic option approved for patients with relapsed/refractory acute lymphoblastic leukemia and diffuse large B-cell lymphoma. Acute kidney injury is a complication of CAR T-cell therapy that can result in kidney failure. In most cases, it is thought to be related to hemodynamic changes due to cytokine release syndrome. Kidney biopsy in this clinical scenario is usually not performed. We report on a kidney transplant recipient in his 40s who developed a posttransplant lymphoproliferative disorder of B-cell origin refractory to conventional treatments and received anti-CD19 CAR T-cell therapy as compassionate treatment. Beginning on day 12 after CAR T-cell infusion, in the absence of clinical symptoms, a progressive decline in estimated glomerular filtration rate of the kidney graft occurred. A subsequent allograft biopsy showed mild tubulointerstitial lymphocyte infiltrates, falling into a Banff borderline-changes category and resembling an acute immunoallergic tubulointerstitial nephritis. Neither CAR T cells nor lymphomatous B cells were detected within the graft cellular infiltrates, suggesting an indirect mechanism of kidney injury. Although kidney graft function partially recovered after steroid therapy, the posttransplant lymphoproliferative disorder progressed and the patient died 7 months later.
\end{abstract}

(C) 2021 The Authors. Published by Elsevier Inc. on behalf of the National Kidney Foundation, Inc. This is an open access article under the CC BY-NC-ND license (http://creativecommons.org/licenses/by-nc-nd/4.0/).

\section{INTRODUCTION}

Posttransplant lymphoproliferative disorder represents a rare but serious complication after organ transplantation. ${ }^{1}$ Treatment includes reduction or withdrawal of immunosuppression followed by rituximab with or without chemotherapy. ${ }^{2,3}$ Transplant recipients who fail to respond or relapse after conventional treatments have a poor prognosis. ${ }^{4}$ Chimeric antigen receptor (CAR) $\mathrm{T}$ cells directed against CD19 surface antigen represent a recent type of autologous adoptive transfer cell therapy currently approved for the treatment of relapsed/refractory diffuse large B-cell lymphoma and other hematologic malignancies. ${ }^{5-8}$ Apart from cytokine release syndrome and neurotoxicity, 9,10 other adverse events have been described, including acute kidney injury (AKI) and electrolyte level abnormalities. ${ }^{11}$ Recently, several solid-organ transplant recipients treated with CAR T-cell therapy were reported. ${ }^{12}$ We describe the case of a kidney transplant recipient who developed severe AKI after CAR T-cell therapy. A kidney biopsy was performed, helping clarify the pathogenesis of this unique case.

\section{CASE REPORT}

A kidney transplant recipient in his 40s was diagnosed with monomorphic type B-cell posttransplant lymphoproliferative disorder approximately 18 years after transplantation; induction therapy included basiliximab. He had a large abdominal mass and supraclavicular lymphadenopathy but no additional organ involvement was detected with staging positron emission tomography computed tomography. He was classified with Ann Arbor stage IVB disease, with an international prognostic score of 2. Histologic characterization of malignant cells showed the following: CD20+, CD79+, CD10+, bcl6+, bcl2+, p53+, c-myc,+ CD3-, and CD5-. Additionally, EpsteinBarr virus-encoded small RNA and human herpesvirus-8 were negative. Fluorescence in situ hybridization was positive for BCL2 but negative for either BCL6 or C-MYC. Ki67 expression was $>80 \%$.

Immunosuppression was reduced, with tacrolimus switched to everolimus and mycophenolate mofetil dose decreased to $50 \%$. Four weekly infusions of rituximab were administered. Due to disease progression, he subsequently received 3 cycles of R-CHOP (rituximab, cyclophosphamide, doxorubicin hydrochloride, vincristine sulfate, and prednisone) immunochemotherapy. Everolimus was reduced to $50 \%$ and mycophenolate mofetil therapy was discontinued. Due to disease persistence, intensification treatment with 2 cycles of R-GDP (rituximab, gemcitabine, dexamethasone, and cisplatin) immunochemotherapy was administered and, with the exception of $5 \mathrm{mg}$ of prednisone daily, immunosuppression was discontinued. Autologous hematopoietic stem cell transplantation consolidation was performed but relapse was observed in positron emission tomography computed tomography performed 3 months after autologous hematopoietic stem cell transplantation. 


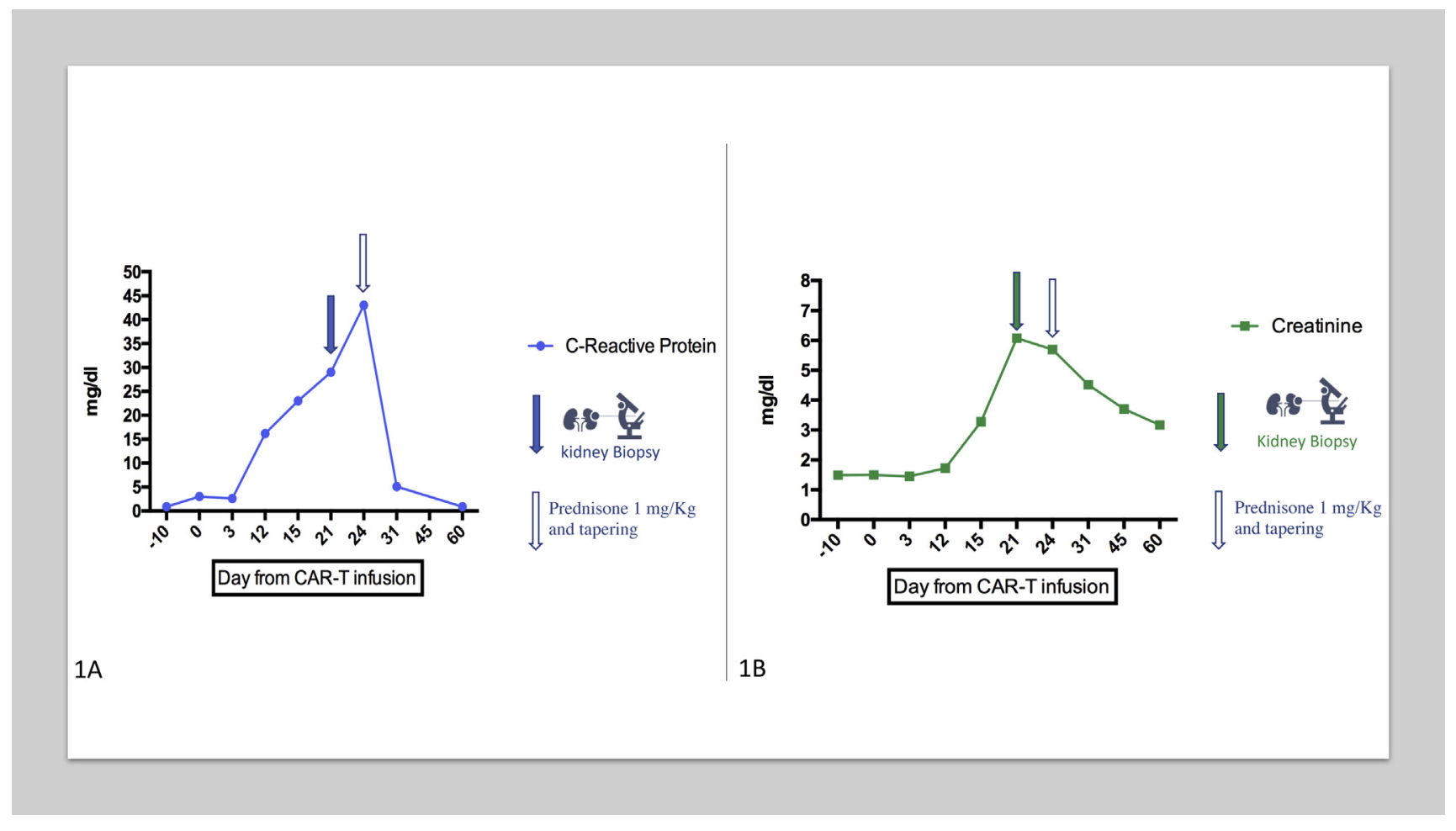

Figure 1. (A) C-Reactive protein and (B) creatinine level evolution during follow-up. Abbreviation: CAR-T, chimeric antigen receptor T-cell.

Approximately 28 months after the initial diagnosis, the patient received lymphodepletion chemotherapy with intravenous fludarabine and intravenous cyclophosphamide for 3 days, followed by CAR T-cell therapy (tisagenlecleucel) infusion. The patient developed grade 3 neutropenia without significant acute complications. Baseline creatinine level was $1.5 \mathrm{mg} / \mathrm{dL}$. At day 12 after CAR T-cell infusion, serum creatinine and C-reactive protein levels began to increase, without relevant symptoms and without oliguria (Fig 1). Neither cytokine release syndrome nor immune effector cell-associated neurotoxicity syndrome were observed. All potentially nephrotoxic drugs (omeprazole and acyclovir) were stopped. Proteinuria was protein excretion of $0.4 \mathrm{~g} / \mathrm{d}$, and sediment showed mild leukocyturia with no erythrocytes. Myoglobin and creatine kinase levels were within the normal range. Polymerase chain reaction for cytomegalovirus was negative. Doppler ultrasound showed a mild increase in kidney echogenicity, with normal resistive index and no sign of obstruction. Calculated panel-reactive antibody level at the time of biopsy was 39\% for the presence of alloantibodies against class II, without donorspecific antibodies.

On day 21 after CAR T-cell therapy administration, serum creatinine level had increased to $6 \mathrm{mg} / \mathrm{dL}$ and a kidney biopsy was performed; C-reactive protein level was $43 \mathrm{mg} / \mathrm{dL}$ at that time. The biopsy showed interstitial fibroedema with moderate mononuclear infiltrates and patchy tubular injury with epithelial thinning, coarse cytoplasmic vacuolization, and mild isolated tubulitis. Arteriolar changes were prominent and demonstrated periodic acid-Schiff-positive hyaline deposits deep in muscle layers. Glomeruli were unremarkable. C4d and immunoglobulins were negative on direct immunofluorescence. Immunohistochemical staining for simian virus 40 was negative. Banff classification was compatible with borderline changes not grading $1 \mathrm{~A}$ acute cellular rejection (Fig S1). The presumptive diagnosis was acute borderline changes in the context of mild alloimmune rejection versus acute immunoallergic interstitial nephritis.

We next aimed to characterize the origin of the lymphocytic infiltrate in the kidney biopsy. As shown in Fig 2, lymphocytic infiltrate was comprised exclusively of CD3+ lymphocytes, with no CD19+ cells. RNA-scope, a technique used to evaluate the expression of the gene encoding the engineered CAR, did not show evidence of CAR Tcell-19 infiltration in the kidney. Aiming to reduce lymphocytic infiltrates but also preserve CAR T-cell function, prednisone was initiated at a dose of $1 \mathrm{mg} / \mathrm{kg}$ per day. ${ }^{13}$ Kidney allograft function partially recovered, reaching a nadir of $3.17 \mathrm{mg} / \mathrm{dL}$ after 60 days (Fig 2), whereas proteinuria was unchanged. Prednisone dosage was progressively tapered to $10 \mathrm{mg}$ per day by 40 days after the kidney biopsy. At 30 and 60 days following CAR T-cell therapy, peripheral-blood CD19+ B-cell counts were undetectable. The patient died approximately 3 years after the initial posttransplant lymphoproliferative disorder diagnosis due to progression of the malignancy. 


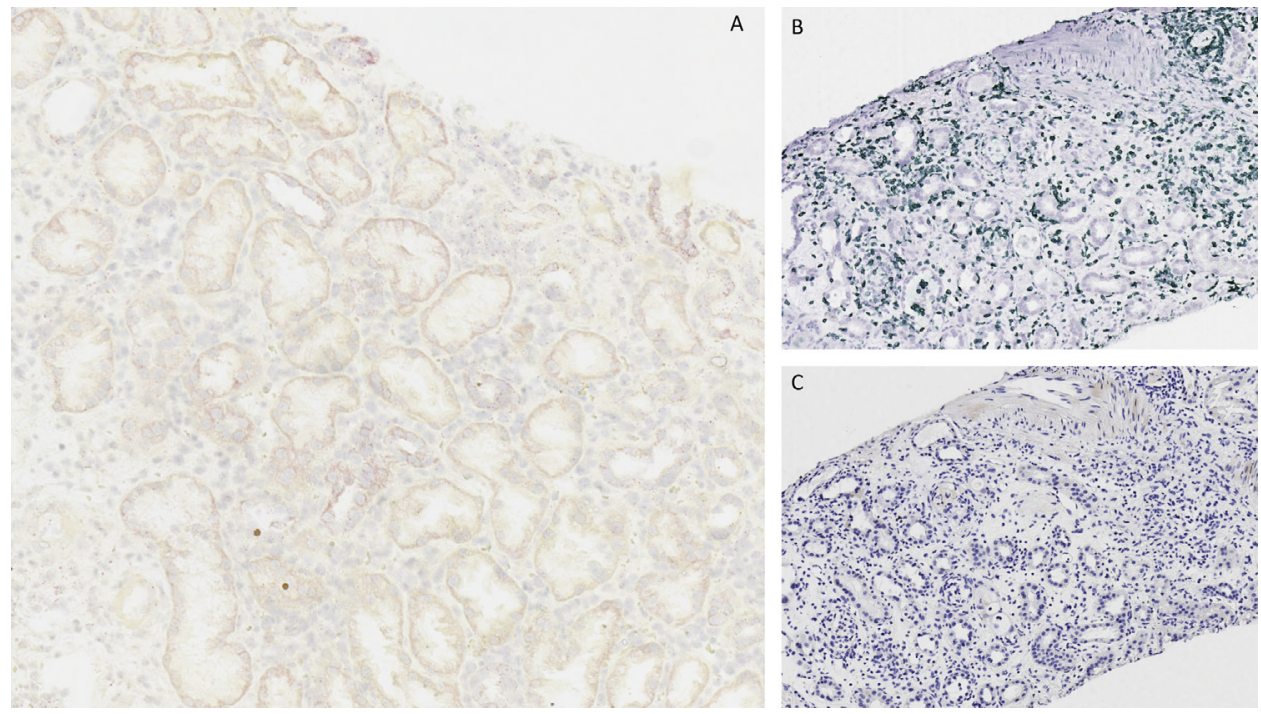

Figure 2. (A) Absence of chimeric antigen receptor (CAR) T cells using RNA scope in situ hybridization. (B) Presence of CD3 cells in kidney tissue. (C) Absence of CD19 cells.

\section{DISCUSSION}

To our knowledge, this is the first report of a kidney biopsy performed in a kidney transplant recipient treated with anti-CD19 CAR T-cell therapy. The main acute treatment-related toxicity was KDIGO (Kidney Disease: Improving Global Outcomes) stage III $\mathrm{AKI}^{14}$ that partially recovered with steroid therapy. AKI has been reported in $19 \%$ to $30 \%$ of patients undergoing CAR T-cell therapy. ${ }^{12,15}$ The occurrence of AKI after CAR T-cell therapy is thought to be functional, secondary to arterial hypotension in the context of cytokine release syndrome. Nonetheless, more severe AKI episodes are likely due to the persistence of severe hemodynamic alterations leading to acute tubular necrosis, ${ }^{16}$ but none of these studies provided data on the histopathology of the kidney injury.

Recently, a study reported that among patients with Bcell lymphoproliferative disorders and kidney lymphoma infiltration, AKI was common. ${ }^{17}$ This observation raises the concern that CAR T-cell therapy might damage the kidney by recognizing kidney tissue-resident B cells. In our case, we did not observe B-cell infiltration in the kidney on a kidney biopsy performed 3 weeks after CAR Tcell therapy infusion. Importantly, no CAR19 RNA was detected, thereby excluding the presence of CAR T-cells in the kidney allograft at the time of the kidney biopsy. Findings observed in the graft histology could have been promoted by a CAR T-cell-induced systemic inflammatory status, thus explaining the resemblance to an acute immunoallergic tubulointerstitial nephritis. Alternatively, biopsy findings could reflect an alloimmune response stimulated by cognate $\mathrm{T}$ cell with endogenous lymphocyte inducing an acute cellular rejection. We decided to use steroids to manage this complication, observing partial recovery of graft function that was sufficient to prevent dialysis therapy and potentially preserve CAR T-cell function, as demonstrated by the absence of peripheralblood CD19+ cells. ${ }^{18}$ Although proton pump inhibitor use has been associated with AKI in patients treated by immune-checkpoint inhibitors ${ }^{19}$ through a second-hit mechanism, no similar data have been reported with CAR T-cell therapy, suggesting that proton pump inhibitor use was not likely the cause of AKI.

In conclusion, although there was no evidence of damage mediated by direct infiltration of CAR T-cells in the kidney, our case suggests that this new therapy could generate kidney graft failure through an indirect inflammatory and/or immune-mediated mechanism, stressing the central role of the kidney biopsy to guide therapy.

\section{SUPPLEMENTARY MATERIAL}

Supplementary File (PDF)

Figure S1. Hematoxylin and eosin stain of kidney tissue. Banff score was compatible with borderline changes (g0, i1-2, ti1-2, i-IFTA1-2, $\mathrm{t} 1, \mathrm{v} 0$, ptc0, ah3, aah3, cg0, ci0, ct0, cv0, and $\mathrm{mm0}$ ).

\section{ARTICLE INFORMATION}

Authors' Full Names and Academic Degrees: Edoardo Melilli, MD, $\mathrm{PhD}$, Alberto Mussetti, MD, Gabriela Sanz Linares, MD, Marco Ruella, MD, PhD, Charette La Salette, PhD, Alexandre Savchenko, $\mathrm{PhD}$, Maria del Rosario Taco, MD, Nuria Montero, MD, PhD, Josep Grinyo, MD, PhD, Alex Fava, MD, Montse Gomà, MD, Maria Meneghini, MD, Anna Manonelles, MD, PhD, Josepmaria Cruzado, MD, PhD, Ana Sureda, MD, PhD, and Oriol Bestard, MD, PhD.

Authors' Affiliations: Nephrology Department, Bellvitge University Hospital-Hospitalet (EM, NM, JG, AF, MM, AManonelles, JC, OB); Institut de Investigació Biomedica de Bellvitge (IDIBELL)Hospitalet (EM, AMussetti, GSL, ASureda, OB); Clinical Hematology Department, Institut Català d'Oncologia-Hospitalet, Barcelona, Spain (AMussetti, GSL, ASureda); Division of Hematology-Oncology, Department of Medicine (MR) and Center for Cellular Immunotherapies (MR), University of Pennsylvania, Philadelphia, PA; IHC/ISH Oncology Precision Medicine, Novartis 
Group (CLS, ASavchenko), Cambridge, MA; and Pathology Department, Bellvitge University Hospital-Hospitalet, Barcelona, Spain (MdRT, MG).

Address for Correspondence: Edoardo Melilli, MD, PhD, Nephrology Department, Bellvitge University Hospital, Feixa llarga s/n 08907, L'Hospitalet de Llobregat, Barcelona, Spain. Email: emelill@bellvitgehospital.cat

Authors' Contributions: Drs Melilli and Mussetti contributed equally to this work.

Support: We thank CERCA Program/Generalitat de Catalunya and the ISCIII RETICS RedinRen RD16/0009/0003 for institutional support.

Financial Disclosure: Dr Mussetti received research funding from Gilead and honoraria for lectures from Novartis. Dr Melilli received research funding from Chiesi and honoraria for lectures from Novartis, Astellas, and Menarini. Dr Ruella has intellectual property in the field of CART immunotherapy that is licensed to Novartis and Tmunity and managed by the University of Pennsylvania and received honoraria and consulting fees by AbClon, BMS, and Nanostring.

Acknowledgements: We are grateful to the patient and his family; during the course of the disease and after the patient's death, they always granted permission to share the patient's history with the scientific community. We thank Novartis Precision Medicine for collaboration in the renal biopsy analysis and imaging.

Patient Protections: The authors declare that they have obtained consent from a relative with appropriate authority for publication of the information about the patient that appears within this Case Report and any associated supplementary material.

Peer Review: Received December 10, 2020, as a submission to the expedited consideration track with 2 external peer reviews. Direct editorial input from an Associate Editor and the Editor-in-Chief. Accepted in revised form March 14, 2021.

\section{REFERENCES}

1. Opelz G, Dohler B. Lymphomas after solid organ transplantation: a collaborative transplant study report. Am J Transplant. 2004;4:222-230.

2. Vikas R, Dharnidharka D. Comprehensive review of post-organ transplant hematologic cancers. Am J Transplant. 2018;18(3): 537-549.

3. Dierickx D, Habermann T. Post-transplantation lymphoproliferative disorders in adults. N Engl J Med. 2018;378(6): 549-562.

4. González-Barca E, Capote FJ, Gómez-Codina J, et al. Longterm follow-up of a prospective phase 2 clinical trial of extended treatment with rituximab in patients with $\mathrm{B}$ cell posttransplant lymphoproliferative disease and validation in real world patients. Ann Hematol. 2021;100(4):1023-1029.
5. Maude SL, Laetsch TW, Buechner J, et al. Tisagenlecleucel in children and young adults with B-cell lymphoblastic leukemia. N Engl J Med. 2018;378(5):439-448.

6. Locke F, Ghobadi A, Jacobson CA, et al. Long-term safety and activity of axicabtagene ciloleucel in refractory large B-cell lymphoma (ZUMA-1): a single-arm, multicentre, phase 1-2 trial. Lancet Oncol. 2019;20(1):31-42.

7. Schuster SJ, Bishop M, Tam C, et al. Tisagenlecleucel in adult relapsed or refractory diffuse large B-cell lymphoma. N Engl J Med. 2019;380:45-56.

8. Wang M, Munoz J, Goy A, et al. KTE-X19 CAR T-cell therapy in relapsed or refractory mantle-cell lymphoma. $N$ Engl J Med. 2020;382:1331-1342.

9. Neelapu S, Tummala S, Kebriae P, et al. Chimeric antigen receptor T-cell therapy-assessment and management of toxicities. Nat Rev Clin Oncol. 2018;15(1):47-62.

10. Brudno J, Kochenderfer JK. Chimeric antigen receptor T-cell therapies for lymphoma. Nat Rev Clin Oncol. 2018;15(1):3146.

11. Gupta S, Seethapathy H, Strohbehn IA, et al. Acute kidney injury and electrolyte abnormalities after chimeric antigen receptor T-cell (CAR-T) therapy for diffuse large B-cell lymphoma. Am J Kidney Dis. 2020;76(1):63-71.

12. Krishnamoorthy S, Ghobadi A, Delos Santos R, et al. CAR-T therapy in solid organ transplant recipients with treatment refractory post transplant lymphoproliferative disorder. Am J Transplant. 2021;21(2):809-814.

13. Bassil C, Khimani F. Immunotherapy use in kidney transplant recipients: immune checkpoint inhibitors and CAR-T cell therapy. J Onconephrol. 2020;4(3):165-170.

14. Aspelin P, Barsoum RS, Burdmann EA, et al. KDIGO clinical practice guideline for acute kidney injury. Accessed January 3, 2021. https://kdigo.org/wpcontent/uploads/2016/10/ KDIGO-2012-AKI-Guideline-English.pdf

15. Gutgarts V, Jain T, Zheng J. Acute kidney injury after CAR-T cell therapy: low incidence and rapid recovery. Biol Blood Marrow Transplant. 2020;26(6):1071-1076.

16. Jhaveri $\mathrm{KD}$, Rosner $\mathrm{MH}$. Chimeric antigen receptor $\mathrm{T}$ cell therapy and the kidney: what the nephrologist needs to know. Clin J Am Soc Nephrol. 2018;13(5):796-798.

17. Javaugue V, Debiais-Delpech $C$, Nouvier $M$, et al. Clinicopathological spectrum of renal parenchymal involvement in Bcell lymphoproliferative disorders. Kidney Int. 2019;96(1):94103.

18. Liu S, Deng B, Yin Z, et al. Corticosteroids do not influence the efficacy and kinetics of CAR-T cells for B-cell acute lymphoblastic leukemia. Blood Cancer J. 2020;10(2):15.

19. Seethapathy H, Zhao S, Chute D, et al. The incidence, causes, and risk factors of acute kidney injury in patients receiving immune checkpoint inhibitors. Clin J Am Soc Nephrol. 2019;14(12):1692-1700. 